\title{
Collapsibility of simplicial complexes of hypergraphs
}

\author{
Alan Lew* \\ Department of Mathematics \\ Technion \\ Haifa 32000, Israel \\ alan@campus.technion.ac.il
}

Submitted: Dec 5, 2018; Accepted: Aug 19, 2019; Published: Oct 11, 2019

(C) The author. Released under the CC BY-ND license (International 4.0).

\begin{abstract}
Let $\mathcal{H}$ be an $r$-uniform hypergraph. We show that the simplicial complex whose simplices are the hypergraphs $\mathcal{F} \subset \mathcal{H}$ with covering number at most $p$ is $\left(\left(\begin{array}{c}r+p \\ r\end{array}\right)-1\right)$ collapsible. Similarly, the simplicial complex whose simplices are the pairwise intersecting hypergraphs $\mathcal{F} \subset \mathcal{H}$ is $\frac{1}{2}\left(\begin{array}{c}2 r \\ r\end{array}\right)$-collapsible.
\end{abstract}

Mathematics Subject Classifications: 05E45, 05D05

\section{Introduction}

Let $X$ be a finite simplicial complex. Let $\eta$ be a simplex of $X$ such that $|\eta| \leqslant d$ and $\eta$ is contained in a unique maximal face $\tau \in X$. We say that the complex

$$
X^{\prime}=X \backslash\{\sigma \in X: \eta \subset \sigma \subset \tau\}
$$

is obtained from $X$ by an elementary d-collapse, and we write $X \stackrel{\eta}{\rightarrow} X^{\prime}$.

The complex $X$ is called $d$-collapsible if there exists a sequence of elementary $d$ collapses

$$
X=X_{1} \stackrel{\eta_{1}}{\longrightarrow} X_{2} \stackrel{\eta_{2}}{\longrightarrow} \cdots \stackrel{\eta_{k-1}}{\longrightarrow} X_{k}=\emptyset
$$

from $X$ to the void complex $\emptyset$. The collapsibility of $X$ is the minimal $d$ such that $X$ is $d$-collapsible.

A simple consequence of $d$-collapsibility is the following:

Proposition 1 (Wegner [11, Lemma 1]). If $X$ is d-collapsible then it is homotopy equivalent to a simplicial complex of dimension smaller than $d$.

\footnotetext{
*Supported by ISF grant no. 326/16.
} 
Let $\mathcal{H}$ be a finite hypergraph. We identify $\mathcal{H}$ with its edge set. The rank of $\mathcal{H}$ is the maximal size of an edge of $\mathcal{H}$.

A set $C$ is a cover of $\mathcal{H}$ if $A \cap C \neq \emptyset$ for all $A \in \mathcal{H}$. The covering number of $\mathcal{H}$, denoted by $\tau(\mathcal{H})$, is the minimal size of a cover of $\mathcal{H}$.

For $p \in \mathbb{N}$, let

$$
\operatorname{Cov}_{\mathcal{H}, p}=\{\mathcal{F} \subset \mathcal{H}: \tau(\mathcal{F}) \leqslant p\} .
$$

That is, $\operatorname{Cov}_{\mathcal{H}, p}$ is a simplicial complex whose vertices are the edges of $\mathcal{H}$ and whose simplices are the hypergraphs $\mathcal{F} \subset \mathcal{H}$ that can be covered by a set of size at most $p$. Some topological properties of the complex $\operatorname{Cov}_{\left(\begin{array}{c}n] \\ r\end{array}\right), p}$ were studied by Jonsson in [6].

The hypergraph $\mathcal{H}$ is called pairwise intersecting if $A \cap B \neq \emptyset$ for all $A, B \in \mathcal{H}$. Let

$$
\operatorname{Int}_{\mathcal{H}}=\{\mathcal{F} \subset \mathcal{H}: A \cap B \neq \emptyset \text { for all } A, B \in \mathcal{F}\} .
$$

So, Int $_{\mathcal{H}}$ is a simplicial complex whose vertices are the edges of $\mathcal{H}$ and whose simplices are the hypergraphs $\mathcal{F} \subset \mathcal{H}$ that are pairwise intersecting.

Our main results are the following:

Theorem 2. Let $\mathcal{H}$ be a hypergraph of rank $r$. Then $\operatorname{Cov}_{\mathcal{H}, p}$ is $\left(\left(\begin{array}{c}r+p \\ r\end{array}\right)-1\right)$-collapsible.

Theorem 3. Let $\mathcal{H}$ be a hypergraph of rank $r$. Then $\operatorname{Int}_{\mathcal{H}}$ is $\frac{1}{2}\left(\begin{array}{c}2 r \\ r\end{array}\right)$-collapsible.

The following examples show that these bounds are sharp:

- Let $\mathcal{H}=\left(\begin{array}{c}{[r+p]} \\ r\end{array}\right)$ be the complete $r$-uniform hypergraph on $r+p$ vertices. The covering number of $\mathcal{H}$ is $p+1$, but for any $A \in \mathcal{H}$ the hypergraph $\mathcal{H} \backslash\{A\}$ can be covered by a set of size $p$, namely by $[r+p] \backslash A$. Therefore the complex $\operatorname{Cov}_{\left(\begin{array}{c}r+p] \\ r\end{array}\right), p}$ is the boundary of the $\left(\left(\begin{array}{c}r+p \\ r\end{array}\right)-1\right)$-dimensional simplex, so it is homeomorphic to a $\left(\left(\begin{array}{c}r+p \\ r\end{array}\right)-2\right)$-dimensional sphere. Hence, by Proposition 1, $\left.\operatorname{Cov}_{(}^{[r+p]}{ }_{r}\right), p$ is not $\left(\left(\begin{array}{c}r+p \\ r\end{array}\right)-2\right)$-collapsible.

- Let $\mathcal{H}=\left(\begin{array}{c}{[2 r]} \\ r\end{array}\right)$ be the complete $r$-uniform hypergraph on $2 r$ vertices. Any $A \in \mathcal{H}$ intersects all the edges of $\mathcal{H}$ except the edge $[2 r] \backslash A$. Therefore the complex $\operatorname{Int}\left(\begin{array}{c}{[2 r]} \\ r\end{array}\right)$ is the boundary of the $\frac{1}{2}\left(\begin{array}{c}2 r \\ r\end{array}\right)$-dimensional cross-polytope, so it is homeomorphic to a $\left(\frac{1}{2}\left(\begin{array}{c}2 r \\ r\end{array}\right)-1\right)$-dimensional sphere. Hence, by Proposition 1, $\operatorname{Int}\left(\begin{array}{c}{[2 r]} \\ r\end{array}\right)$ is not $\left(\frac{1}{2}\left(\begin{array}{c}2 r \\ r\end{array}\right)-1\right)$ collapsible.

A related problem was studied by Aharoni, Holzman and Jiang in [2], where they show that for any $r$-uniform hypergraph $\mathcal{H}$ and $p \in \mathbb{Q}$, the complex of hypergraphs $\mathcal{F} \subset \mathcal{H}$ with fractional matching number (or equivalently, fractional covering number) smaller than $p$ is $(\lceil r p\rceil-1)$-collapsible.

Our proofs rely on two main ingredients. The first one is the following theorem:

Theorem 4. Let $X$ be a simplicial complex on vertex set $V$. Let $S(X)$ be the collection of all sets $\left\{v_{1}, \ldots, v_{k}\right\} \subset V$ satisfying the following condition: 
There exist maximal faces $\sigma_{1}, \sigma_{2}, \ldots, \sigma_{k+1}$ of $X$ such that:

- $v_{i} \notin \sigma_{i}$ for all $i \in[k]$,

- $v_{i} \in \sigma_{j}$ for all $1 \leqslant i<j \leqslant k+1$.

Let $d^{\prime}(X)$ be the maximum size of a set in $S(X)$. Then $X$ is $d^{\prime}(X)$-collapsible.

Theorem 4 is a special case of a more general result, due essentially to Matoušek and Tancer (who stated it in the special case where the complex is the nerve of a family of finite sets, and used it to prove the case $p=1$ of Theorem 2; see [9]).

The second ingredient is the following combinatorial lemma, proved independently by Frankl and Kalai.

Lemma 5 (Frankl [4], Kalai [7]). Let $\left\{A_{1}, \ldots, A_{k}\right\}$ and $\left\{B_{1}, \ldots, B_{k}\right\}$ be families of sets such that:

- $\left|A_{i}\right| \leqslant r,\left|B_{i}\right| \leqslant p$ for all $i \in[k]$,

- $A_{i} \cap B_{i}=\emptyset$ for all $i \in[k]$,

- $A_{i} \cap B_{j} \neq \emptyset$ for all $1 \leqslant i<j \leqslant k$.

Then

$$
k \leqslant\left(\begin{array}{c}
r+p \\
r
\end{array}\right) .
$$

The paper is organized as follows. In Section 2 we present Matoušek and Tancer's bound on the collapsibility of a simplicial complex, and we prove Theorem 4. In Section 3 we present some results on the collapsibility of independence complexes of graphs. In Section 4 we prove our main results on the collapsibility of complexes of hypergraphs. Section 5 contains some generalizations of Theorems 2 and 3, which are obtained by applying different known variants of Lemma 5.

\section{A bound on the collapsibility of a complex}

Let $X$ be a (non-void) simplicial complex on vertex set $V$. Fix a linear order $<$ on $V$. Let $\mathcal{A}=\left(\sigma_{1}, \ldots, \sigma_{m}\right)$ be a sequence of faces of $X$ such that, for any $\sigma \in X, \sigma \subset \sigma_{i}$ for some $i \in[m]$. For example, we may take $\sigma_{1}, \ldots, \sigma_{m}$ to be the set of maximal faces of $X$ (ordered in any way).

For a simplex $\sigma \in X$, let $m_{X, \mathcal{A},<}(\sigma)=\min \left\{i \in[m]: \sigma \subset \sigma_{i}\right\}$. Let $i \in[m]$ and $\sigma \in X$ such that $m_{X, \mathcal{A},<}(\sigma)=i$. We define the minimal exclusion sequence

$$
\operatorname{mes}_{X, \mathcal{A},<}(\sigma)=\left(v_{1}, \ldots, v_{i-1}\right)
$$

as follows: If $i=1$ then $\operatorname{mes}_{X, \mathcal{A},<}(\sigma)$ is the empty sequence. If $i>1$ we define the sequence recursively as follows: 
Since $i>1$, we must have $\sigma \not \subset \sigma_{1}$; hence, there is some $v \in \sigma$ such that $v \notin \sigma_{1}$. Let $v_{1}$ be the minimal such vertex (with respect to the order $<$ ).

Let $1<j<i$ and assume that we already defined $v_{1}, \ldots, v_{j-1}$. Since $i>j$, we must have $\sigma \not \subset \sigma_{j}$; hence, there exists some $v \in \sigma$ such that $v \notin \sigma_{j}$.

- If there is a vertex $v_{k} \in\left\{v_{1}, \ldots, v_{j-1}\right\}$ such that $v_{k} \notin \sigma_{j}$, let $v_{j}$ be such a vertex of minimal index $k$. In this case we call $v_{j}$ old at $j$.

- If $v_{k} \in \sigma_{j}$ for all $k<j$, let $v_{j}$ be the minimal vertex $v \in \sigma$ (with respect to the order $<)$ such that $v \notin \sigma_{j}$. In this case we call $v_{j}$ new at $j$.

Let $M_{X, \mathcal{A},<}(\sigma) \subset \sigma$ be the simplex consisting of all the vertices appearing in the sequence $\operatorname{mes}_{X, \mathcal{A},<}(\sigma)$. Let

$$
d(X, \mathcal{A},<)=\max \left\{\left|M_{X, \mathcal{A},<}(\sigma)\right|: \sigma \in X\right\} .
$$

The following result was stated and proved in [9, Prop. 1.3] in the special case where $X$ is the nerve of a finite family of sets (in our notation, $X=\operatorname{Cov}_{\mathcal{H}, 1}$ for some hypergraph $\mathcal{H})$.

Theorem 6. The simplicial complex $X$ is $d(X, \mathcal{A},<)$-collapsible.

The proof given in [9] can be easily modified to hold in this more general setting. Here we present a different proof.

Let $X$ be a simplicial complex on vertex set $V$, and let $v \in V$. Let

$$
X \backslash v=\{\sigma \in X: v \notin \sigma\}
$$

and

$$
\operatorname{lk}(X, v)=\{\sigma \in X: v \notin \sigma, \sigma \cup\{v\} \in X\} .
$$

We will need the following lemma, proved by Tancer in [10]:

Lemma 7 (Tancer [10, Prop. 1.2]). If $X \backslash v$ is d-collapsible and $\operatorname{lk}(X, v)$ is $(d-1)$ collapsible, then $X$ is d-collapsible.

Proof of Theorem 6. First, we deal with the case where $X$ is a complete complex (i.e. a simplex). Then $X$ is 0-collapsible; therefore, the claim holds.

For a general complex $X$, we argue by induction on the number of vertices of $X$. If $|V|=0$, then $X=\{\emptyset\}$. In particular, it is a complete complex; hence, the claim holds.

Let $|V|>0$, and assume that the claim holds for any complex with less than $|V|$ vertices. If $\sigma_{1}=V$, then $X$ is the complete complex on vertex set $V$, and the claim holds. Otherwise, let $v$ be the minimal vertex (with respect to $<$ ) in $V \backslash \sigma_{1}$.

In order to apply Lemma 7, we will need the following two claims:

Claim 8. The complex $X \backslash v$ is $d(X, \mathcal{A},<)$-collapsible. 
Proof. For every $i \in[m]$, let $\sigma_{i}^{\prime}=\sigma_{i} \backslash\{v\}$, and let $\mathcal{A}^{\prime}=\left(\sigma_{1}^{\prime}, \ldots, \sigma_{m}^{\prime}\right)$. Let $\sigma \in X \backslash v$. Since $v \notin \sigma$, then, for any $i \in[m], \sigma \subset \sigma_{i}$ if and only if $\sigma \subset \sigma_{i}^{\prime}$. Hence, every simplex $\sigma \in X \backslash v$ is contained in $\sigma_{i}^{\prime}$ for some $i \in[m]$ (since, by the definition of $\mathcal{A}, \sigma \subset \sigma_{i}$ for some $i \in[m])$. So, by the induction hypothesis, $X \backslash v$ is $d\left(X \backslash v, \mathcal{A}^{\prime},<\right)$-collapsible.

Let $\sigma \in X \backslash v$. We will show that $\operatorname{mes}_{X, \mathcal{A},<}(\sigma)=\operatorname{mes}_{X \backslash v, \mathcal{A}^{\prime},<}(\sigma)$. Since for any $i \in[m]$, $\sigma \subset \sigma_{i}$ if and only if $\sigma \subset \sigma_{i}^{\prime}$, then the two sequences are of the same length. Let

$$
\operatorname{mes}_{X, \mathcal{A},<}(\sigma)=\left(v_{1}, \ldots, v_{k}\right)
$$

and

$$
\operatorname{mes}_{X \backslash v, \mathcal{A}^{\prime},<}(\sigma)=\left(v_{1}^{\prime}, \ldots, v_{k}^{\prime}\right) .
$$

We will show that $v_{i}=v_{i}^{\prime}$ for all $i \in[k]$. We argue by induction on $i$. Let $i \in[k]$, and assume that $v_{j}=v_{j}^{\prime}$ for all $j<i$. Since $v \notin \sigma$, then $\sigma \backslash \sigma_{i}=\sigma \backslash \sigma_{i}^{\prime}$. Therefore, for any $j<i, v_{j} \in \sigma \backslash \sigma_{i}$ if and only if $v_{j}^{\prime}=v_{j} \in \sigma \backslash \sigma_{i}^{\prime}$. Hence, $v_{i}$ is old at $i$ if and only if $v_{i}^{\prime}$ is old at $i$, and if $v_{i}$ and $v_{i}^{\prime}$ are both old at $i$, then $v_{i}=v_{i}^{\prime}$. Otherwise, both $v_{i}$ and $v_{i}^{\prime}$ are new at $i$. Then, $v_{i}$ is the minimal vertex in $\sigma \backslash \sigma_{i}$, and $v_{i}^{\prime}$ is the minimal vertex in $\sigma \backslash \sigma_{i}^{\prime}=\sigma \backslash \sigma_{i}$. Thus, $v_{i}=v_{i}^{\prime}$.

Therefore, $\left|M_{X \backslash v, \mathcal{A}^{\prime},<}(\sigma)\right|=\left|M_{X, \mathcal{A},<}(\sigma)\right|$ for any $\sigma \in X \backslash v$; hence,

$$
d\left(X \backslash v, \mathcal{A}^{\prime},<\right) \leqslant d(X, \mathcal{A},<) .
$$

So, $X \backslash v$ is $d(X, \mathcal{A},<)$-collapsible.

Claim 9. The complex $\operatorname{lk}(X, v)$ is $(d(X, \mathcal{A},<)-1)$-collapsible.

Proof. Let $I=\left\{i \in[m]: v \in \sigma_{i}\right\}$. For every $i \in I$, let $\sigma_{i}^{\prime \prime}=\sigma_{i} \backslash\{v\}$. Write $I=\left\{i_{1}, \ldots, i_{r}\right\}$, where $i_{1}<\cdots<i_{r}$, and let $\mathcal{A}^{\prime \prime}=\left(\sigma_{i_{1}}^{\prime \prime}, \ldots, \sigma_{i_{r}}^{\prime \prime}\right)$.

For any $\sigma \in \operatorname{lk}(X, v)$, the simplex $\sigma \cup\{v\}$ belongs to $X$; hence, there exists some $i \in[m]$ such that $\sigma \cup\{v\} \subset \sigma_{i}$. Since $v \in \sigma \cup\{v\}$, we must have $i \in I$, and therefore $\sigma \subset$ $\sigma_{i}^{\prime \prime}=\sigma_{i} \backslash\{v\}$. So, by the induction hypothesis, $\operatorname{lk}(X, v)$ is $d\left(\operatorname{lk}(X, v), \mathcal{A}^{\prime \prime},<\right)$-collapsible.

Let $\sigma \in \operatorname{lk}(X, v)$. We will show that

$$
M_{X, \mathcal{A},<}(\sigma \cup\{v\})=M_{\mathrm{lk}(X, v), \mathcal{A}^{\prime \prime},<}(\sigma) \cup\{v\} .
$$

Let

$$
\operatorname{mes}_{X, \mathcal{A},<}(\sigma \cup\{v\})=\left(v_{1}, \ldots, v_{n}\right),
$$

and

$$
\operatorname{mes}_{\operatorname{lk}(X, v), \mathcal{A}^{\prime \prime},<}(\sigma)=\left(u_{1}, \ldots, u_{t}\right) .
$$

For any $j \in[r], \sigma \subset \sigma_{i_{j}}^{\prime \prime}$ if and only if $\sigma \cup\{v\} \subset \sigma_{i_{j}}$. Also, for $i \notin I, \sigma \cup\{v\} \not \subset \sigma_{i}$ (since $\left.v \notin \sigma_{i}\right)$. Therefore, $n=i_{t+1}-1$.

The vertex $v$ is the minimal vertex in $V \backslash \sigma_{1}$, therefore it is the minimal vertex in $(\sigma \cup\{v\}) \backslash \sigma_{1}$. Hence, we have $v_{1}=v$. Now, let $i>1$ such that $i \notin I$. Then, $v_{1}=v$ is the vertex of minimal index in the sequence $\left(v_{1}, \ldots, v_{i-1}\right)$ that is contained in $(\sigma \cup\{v\}) \backslash \sigma_{i}$. Therefore, $v_{i}=v$. 
Finally, we will show that $v_{i_{j}}=u_{j}$ for all $j \in[t]$. We argue by induction on $j$. Let $j \in[t]$, and assume that $v_{i_{\ell}}=u_{\ell}$ for all $\ell<j$.

For any $k<i_{j}$, either $v_{k}=v$ (if $k \notin I$ ) or $v_{k}=u_{\ell}$ for some $\ell<j$ (if $k=i_{\ell} \in I$ ). Also, since $v \in \sigma_{i_{j}}$, we have $(\sigma \cup\{v\}) \backslash \sigma_{i_{j}}=\sigma \backslash \sigma_{i_{j}}^{\prime \prime}$. So, for any $k<i_{j}, v_{k} \in(\sigma \cup\{v\}) \backslash \sigma_{i_{j}}$ if and only if $k=i_{\ell}$ for some $\ell<j$ such that $u_{\ell} \in \sigma \backslash \sigma_{i_{j}}^{\prime \prime}$. Therefore, $v_{i_{j}}$ is old at $i_{j}$ if and only if $u_{j}$ is old at $j$, and if $v_{i_{j}}$ and $u_{j}$ are both old, then $v_{i_{j}}=u_{j}$. Otherwise, assume that $v_{i_{j}}$ is new at $i_{j}$ and $u_{j}$ is new at $j$. Then, $v_{i_{j}}$ is the minimal vertex in $(\sigma \cup\{v\}) \backslash \sigma_{i_{j}}$, and $u_{j}$ is the minimal vertex in $\sigma \backslash \sigma_{i_{j}}^{\prime \prime}=(\sigma \cup\{v\}) \backslash \sigma_{i_{j}}$. Thus, $v_{i_{j}}=u_{j}$.

So, for any $\sigma \in \operatorname{lk}(X, v)$ we obtain

$$
\left|M_{\mathrm{lk}(X, v), \mathcal{A}^{\prime \prime},<}(\sigma)\right|=\left|M_{X, \mathcal{A},<}(\sigma \cup\{v\})\right|-1 .
$$

Hence,

$$
d\left(\operatorname{lk}(X, v), \mathcal{A}^{\prime \prime},<\right) \leqslant d(X, \mathcal{A},<)-1 .
$$

So, $\operatorname{lk}(X, v)$ is $(d(X, \mathcal{A},<)-1)$-collapsible.

By Claim 8, Claim 9 and Lemma $7, X$ is $d(X, \mathcal{A},<)$-collapsible.

Proof of Theorem 4. Let $<$ be some linear order on the vertex set $V$, and let $\mathcal{A}=$ $\left(\sigma_{1}, \ldots, \sigma_{m}\right)$ be the sequence of maximal faces of $X$ (ordered in any way).

Let $i \in[m]$ and let $\sigma \in X$ with $m_{X, \mathcal{A},<}(\sigma)=i$. Let $\operatorname{mes}_{X, \mathcal{A},<}(\sigma)=\left(v_{1}, \ldots, v_{i-1}\right)$. Then $M_{X, \mathcal{A},<}(\sigma)=\left\{v_{i_{1}}, \ldots, v_{i_{k}}\right\}$ for some $i_{1}<\cdots<i_{k} \in[i-1]$ (these are exactly the indices $i_{j}$ such that $v_{i_{j}}$ is new at $i_{j}$ ). For each $j \in[k]$ we have $v_{i_{j}} \notin \sigma_{i_{j}}$. In addition, since $v_{i_{j}}$ is new at $i_{j}$, we have $v_{i_{\ell}} \in \sigma_{i_{j}}$ for all $\ell<j$. Let $i_{k+1}=i$. Since $m_{X, \mathcal{A},<}(\sigma)=i=i_{k+1}$, we have $\sigma \subset \sigma_{i_{k+1}}$. In particular, $v_{i_{\ell}} \in \sigma_{i_{k+1}}$ for all $\ell<k+1$.

Therefore, $M_{X, \mathcal{A},<}(\sigma) \in S(X)$. Thus, $d(X, \mathcal{A},<) \leqslant d^{\prime}(X)$, and by Theorem $6, X$ is $d^{\prime}(X)$-collapsible.

\section{Collapsibility of independence complexes}

Let $G=(V, E)$ be a graph. The independence complex $I(G)$ is the simplicial complex on vertex set $V$ whose simplices are the independent sets in $G$.

Definition 10. Let $k(G)$ be the maximal size of a set $\left\{v_{1}, \ldots, v_{k}\right\} \subset V$ that satisfies:

- $\left\{v_{i}, v_{j}\right\} \notin E$ for all $i \neq j \in[k]$,

- There exist $u_{1}, \ldots, u_{k} \in V$ such that

- $\left\{v_{i}, u_{i}\right\} \in E$ for all $i \in[k]$,

$-\left\{v_{i}, u_{j}\right\} \notin E$ for all $1 \leqslant i<j \leqslant k$.

Proposition 11. $k(G)=d^{\prime}(I(G))$.

Proof. Let $A=\left\{v_{1}, \ldots, v_{k}\right\} \in S(I(G))$. Then, there exist maximal faces $\sigma_{1}, \ldots, \sigma_{k+1}$ of $I(G)$ such that: 
- $v_{i} \notin \sigma_{i}$ for all $i \in[k]$,

- $v_{i} \in \sigma_{j}$ for all $1 \leqslant i<j \leqslant k+1$.

Let $i \in[k]$. Since $\sigma_{i}$ is a maximal independent set in $G$ and $v_{i} \notin \sigma_{i}$, there exists some $u_{i} \in \sigma_{i}$ such that $\left\{v_{i}, u_{i}\right\} \in E$.

Let $1 \leqslant i<j \leqslant k$. Since $v_{i}$ and $u_{j}$ are both contained in the independent set $\sigma_{j}$, we have $\left\{v_{i}, u_{j}\right\} \notin E$. Furthermore, since $A \subset \sigma_{k+1}, A$ is an independent set in $G$. That is, $\left\{v_{i}, v_{j}\right\} \notin E$ for all $i \neq j \in[k]$. So, $A$ satisfies the conditions of Definition 10. Hence, $|A| \leqslant k(G)$; therefore, $d^{\prime}(I(G)) \leqslant k(G)$.

Now, let $k=k(G)$, and let $v_{1}, \ldots, v_{k}, u_{1}, \ldots, u_{k} \in V$ such that

- $\left\{v_{i}, v_{j}\right\} \notin E$ for all $i \neq j \in[k]$,

- $\left\{v_{i}, u_{i}\right\} \in E$ for all $i \in[k]$,

- $\left\{v_{i}, u_{j}\right\} \notin E$ for all $1 \leqslant i<j \leqslant k$.

Let $i \in[k]$, and let $V_{i}=\left\{v_{j}: 1 \leqslant j<i\right\}$. Note that $V_{i} \cup\left\{u_{i}\right\}$ forms an independent set in $G$; therefore, it is a simplex in $I(G)$. Let $\sigma_{i}$ be a maximal face of $I(G)$ containing $V_{i} \cup\left\{u_{i}\right\}$. Since $\left\{v_{i}, u_{i}\right\} \in E$, we have $v_{i} \notin \sigma_{i}$.

The set $\left\{v_{1}, \ldots, v_{k}\right\}$ is also an independent set in $G$. Therefore, there is a maximal face $\sigma_{k+1} \in I(G)$ that contains it.

By the definition of $\sigma_{1}, \ldots, \sigma_{k+1}$, we have $v_{i} \in \sigma_{j}$ for $1 \leqslant i<j \leqslant k+1$. Therefore, $\left\{v_{1}, \ldots, v_{k}\right\} \in S(I(G))$; so, $k(G)=k \leqslant d^{\prime}(I(G))$. Hence, $k(G)=d^{\prime}(I(G))$, as wanted.

As an immediate consequence of Proposition 11 and Theorem 4, we obtain:

Proposition 12. The complex $I(G)$ is $k(G)$-collapsible.

Note that vertices $v_{1}, \ldots, v_{k}, u_{1}, \ldots, u_{k} \in V$ satisfying the conditions in Definition 10 must all be distinct. As a simple corollary, we obtain

Corollary 13. The independence complex of a graph $G=(V, E)$ on $n$ vertices is $\left\lfloor\frac{n}{2}\right\rfloor$ collapsible.

\section{Complexes of hypergraphs}

In this section we prove our main results, Theorems 2 and 3.

Proof of Theorem 2. Let $\mathcal{H}$ be a hypergraph of rank $r$ on vertex set $[n]$, and let

$$
\left\{A_{1}, \ldots, A_{k}\right\} \in S\left(\operatorname{Cov}_{\mathcal{H}, p}\right) \text {. }
$$

Then, there exist maximal faces $\mathcal{F}_{1}, \ldots, \mathcal{F}_{k+1} \in \mathrm{Cov}_{\mathcal{H}, p}$ such that

- $A_{i} \notin \mathcal{F}_{i}$ for all $i \in[k]$, 
- $A_{i} \in \mathcal{F}_{j}$ for all $1 \leqslant i<j \leqslant k+1$.

For any $i \in[k+1]$, there is some $C_{i} \subset[n]$ of size at most $p$ that covers $\mathcal{F}_{i}$. Since $\mathcal{F}_{i}$ is maximal, then, for any $A \in \mathcal{H}, A \in \mathcal{F}_{i}$ if and only if $A \cap C_{i} \neq \emptyset$. Therefore, we obtain

- $A_{i} \cap C_{i}=\emptyset$ for all $i \in[k]$,

- $A_{i} \cap C_{j} \neq \emptyset$ for all $1 \leqslant i<j \leqslant k+1$.

Hence, the pair of families

$$
\left\{A_{1}, \ldots A_{k}, \emptyset\right\}
$$

and

$$
\left\{C_{1}, \ldots, C_{k}, C_{k+1}\right\}
$$

satisfies the conditions of Lemma 5 ; thus, $k+1 \leqslant\left(\begin{array}{c}r+p \\ r\end{array}\right)$. Therefore,

$$
d^{\prime}\left(\operatorname{Cov}_{\mathcal{H}, p}\right) \leqslant\left(\begin{array}{c}
r+p \\
r
\end{array}\right)-1
$$

and by Theorem $4, \operatorname{Cov}_{\mathcal{H}, p}$ is $\left(\left(\begin{array}{c}r+p \\ r\end{array}\right)-1\right)$-collapsible.

Proof of Theorem 3. Let $\mathcal{H}$ be a hypergraph of rank $r$ and let $G$ be the graph on vertex set $\mathcal{H}$ whose edges are the pairs $\{A, B\} \subset \mathcal{H}$ such that $A \cap B=\emptyset$. Then $\operatorname{Int}_{\mathcal{H}}=I(G)$.

Let $k=k(G)$ and let $\left\{A_{1}, \ldots, A_{k}\right\} \subset \mathcal{H}$ that satisfies the conditions of Definition 10 . That is,

- $A_{i} \cap A_{j} \neq \emptyset$ for all $i \neq j \in[k]$,

- There exist $B_{1}, \ldots, B_{k} \in \mathcal{H}$ such that

$$
\begin{aligned}
& -A_{i} \cap B_{i}=\emptyset \text { for all } i \in[k], \\
& -A_{i} \cap B_{j} \neq \emptyset \text { for all } 1 \leqslant i<j \leqslant k .
\end{aligned}
$$

Then, the pair of families $\left\{A_{1}, \ldots, A_{k}, B_{k}, \ldots, B_{1}\right\}$ and $\left\{B_{1}, \ldots, B_{k}, A_{k}, \ldots, A_{1}\right\}$ satisfies the conditions of Lemma 5 ; therefore, $2 k \leqslant\left(\begin{array}{c}2 r \\ r\end{array}\right)$. Thus, by Proposition $12, \operatorname{Int}_{\mathcal{H}}=I(G)$ is $\frac{1}{2}\left(\begin{array}{c}2 r \\ r\end{array}\right)$-collapsible.

\section{More complexes of hypergraphs}

Let $\mathcal{H}$ be a hypergraph. A set $C$ is a $t$-transversal of $\mathcal{H}$ if $|A \cap C| \geqslant t$ for all $A \in \mathcal{H}$. Let $\tau_{t}(\mathcal{H})$ be the minimal size of a $t$-transversal of $\mathcal{H}$. The hypergraph $\mathcal{H}$ is pairwise t-intersecting if $|A \cap B| \geqslant t$ for all $A, B \in \mathcal{H}$. Let

$$
\operatorname{Cov}_{\mathcal{H}, p}^{t}=\left\{\mathcal{F} \subset \mathcal{H}: \tau_{t}(\mathcal{F}) \leqslant p\right\}
$$

and

$$
\operatorname{Int}_{\mathcal{H}}^{t}=\{\mathcal{F} \subset \mathcal{H}: \mathcal{F} \text { is pairwise } t \text {-intersecting }\} .
$$

The following generalization of Lemma 5 was proved by Füredi in [5]. 
Lemma 14 (Füredi [5]). Let $\left\{A_{1}, \ldots, A_{k}\right\}$ and $\left\{B_{1}, \ldots, B_{k}\right\}$ be families of sets such that:

- $\left|A_{i}\right| \leqslant r,\left|B_{i}\right| \leqslant p$ for all $i \in[k]$,

- $\left|A_{i} \cap B_{i}\right| \leqslant t$ for all $i \in[k]$,

- $\left|A_{i} \cap B_{j}\right|>t$ for all $1 \leqslant i<j \leqslant k$.

Then

$$
k \leqslant\left(\begin{array}{c}
r+p-2 t \\
r-t
\end{array}\right)
$$

We obtain the following:

Theorem 15. Let $\mathcal{H}$ be a hypergraph of rank $r$ and let $t \leqslant \min \{r, p\}-1$. Then $\operatorname{Cov}_{\mathcal{H}, p}^{t+1}$ is $\left(\left(\begin{array}{c}r+p-2 t \\ r-t\end{array}\right)-1\right)$-collapsible.

Theorem 16. Let $\mathcal{H}$ be a hypergraph of rank $r$ and let $t \leqslant r-1$. Then $\operatorname{Int}_{\mathcal{H}}^{t+1}$ is $\frac{1}{2}\left(\begin{array}{c}2(r-t) \\ r-t\end{array}\right)$ collapsible.

Note that by setting $t=0$ we recover Theorems 2 and 3 . The proofs are essentially the same as the proofs of Theorems 2 and 3, except for the use of Lemma 14 instead of Lemma 5. The extremal examples are also similar: Let

$$
\mathcal{H}_{1}=\left\{A \cup[t]: A \in\left(\begin{array}{c}
{[r+p-t] \backslash[t]} \\
r-t
\end{array}\right)\right\}
$$

and

$$
\mathcal{H}_{2}=\left\{A \cup[t]: A \in\left(\begin{array}{c}
{[2 r-t] \backslash[t]} \\
r-t
\end{array}\right)\right\}
$$

The complex $\operatorname{Cov}_{\mathcal{H}_{1}}^{t+1}$ is the boundary of the $\left(\left(\begin{array}{c}r+p-2 t \\ r-t\end{array}\right)-1\right)$-dimensional simplex, hence it is not $\left(\left(\begin{array}{c}r+p-2 t \\ r-t\end{array}\right)-2\right)$-collapsible, and the complex $\operatorname{Int}_{\mathcal{H}_{2}}^{t+1}$ is the boundary of the $\frac{1}{2}\left(\begin{array}{c}2(r-t) \\ r-t\end{array}\right)$ dimensional cross-polytope, hence it is not $\left(\frac{1}{2}\left(\begin{array}{c}2(r-t) \\ r-t\end{array}\right)-1\right)$-collapsible.

Restricting ourselves to special classes of hypergraphs we may obtain better bounds on the collapsibility of their associated complexes. For example, we may look at $r$-partite $r$-uniform hypergraphs (that is, hypergraphs $\mathcal{H}$ on vertex set $V=V_{1} \bullet V_{2} \bullet \cdots \bullet V_{r}$ such that $\left|A \cap V_{i}\right|=1$ for all $A \in \mathcal{H}$ and $\left.i \in[r]\right)$. In this case we have the following result:

Theorem 17. Let $\mathcal{H}$ be an $r$-partite $r$-uniform hypergraph. Then $\operatorname{Int}_{\mathcal{H}}$ is $2^{r-1}$-collapsible.

The next example shows that the bound on the collapsibility of $\operatorname{Int}_{\mathcal{H}}$ in Theorem 17 is tight: Let $\mathcal{H}$ be the complete $r$-partite $r$-uniform hypergraph with all sides of size 2 . It has $2^{r}$ edges, and any edge $A \in \mathcal{H}$ intersects all the edges of $\mathcal{H}$ except its complement. Therefore the complex $\operatorname{Int}_{\mathcal{H}}$ is the boundary of the $2^{r-1}$-dimensional cross-polytope, so it is homeomorphic to a $\left(2^{r-1}-1\right)$-dimensional sphere. Hence, by Proposition $1, \operatorname{Int}_{\mathcal{H}}$ is not $\left(2^{r-1}-1\right)$-collapsible.

For the proof we need the following Lemma, due to Lovász, Nešetřil and Pultr. 
Lemma 18 (Lovász, Nešetřil, Pultr [8, Prop. 5.3]). Let $\left\{A_{1}, \ldots, A_{k}\right\}$ and $\left\{B_{1}, \ldots, B_{k}\right\}$ be families of subsets of $V=V_{1} \cup V_{2} \bullet \cdots \bullet V_{r}$ such that:

- $\left|A_{i} \cap V_{j}\right|=1,\left|B_{i} \cap V_{j}\right|=1$ for all $i \in[k]$ and $j \in[r]$,

- $A_{i} \cap B_{i}=\emptyset$ for all $i \in[k]$,

- $A_{i} \cap B_{j} \neq \emptyset$ for all $1 \leqslant i<j \leqslant k$.

Then $k \leqslant 2^{r}$.

A common generalization of Lemma 5 and Lemma 18 was proved by Alon in [3].

The proof of Theorem 17 is the same as the proof of Theorem 3, except that we replace Lemma 5 by Lemma 18. A similar argument was also used by Aharoni and Berger ([1, Theorem 5.1]) in order to prove a related result about rainbow matchings in $r$-partite $r$-uniform hypergraphs.

\section{Acknowledgements}

I thank Professor Roy Meshulam for his guidance and help. I thank the anonymous referee for some helpful suggestions.

\section{References}

[1] R. Aharoni and E. Berger. Rainbow matchings in $r$-partite $r$-graphs. Electronic $J$. Combinatorics, 16(1):119, 2009.

[2] R. Aharoni, R. Holzman, and Z. Jiang. Rainbow fractional matchings. Preprint, arxiv: 1805.09732, 2018.

[3] N. Alon. An extremal problem for sets with applications to graph theory. J. Comb. Theory (A), 40(1):82-89, 1985.

[4] P. Frankl. An extremal problem for two families of sets. Eur. J. Comb., 3(2):125-127, 1982.

[5] Z. Füredi. Geometrical solution of an intersection problem for two hypergraphs. Eur. J. Comb., 5(2):133-136, 1984.

[6] J. Jonsson. Simplicial complexes of graphs. Lecture Notes in Math. 1928, Springer, Heidelberg, 2008.

[7] G. Kalai. Intersection patterns of convex sets. Israel J. Math., 48(2-3):161-174, 1984.

[8] L. Lovász, J. Nešetřil, and A. Pultr. On a product dimension of graphs. J. Comb. Theory (B), 29(1):47-67, 1980.

[9] J. Matoušek and M. Tancer. Dimension gaps between representability and collapsibility. Discrete Comput. Geom., 42(4):631-639, 2009.

[10] M. Tancer. Strong d-collapsibility. Contributions to Discrete Mathematics, 6(2):3235, 2011.

[11] G. Wegner. d-collapsing and nerves of families of convex sets. Arch. Math., 26(1):317321, 1975. 